\title{
Reconstructive Surgery Of Congenital And Acquired Iris Defects Carried Out Between 2012 And 2019 At A Private Medical Center In Poland
}

\author{
A. Cywinski
}

\begin{abstract}
Retrospective evaluation of the effects of surgical treatment of congenital and acquired iris defects. Procedures were performed by one surgeon in 47 patients, in 53 eyes. In the case of six eyes (three eyes were blind), a cosmetic procedure was performed. In some cases, iris implants were used. Congenital partial iris loss (coloboma) was treated using the author's proprietary surgical technique. In many cases, iris surgery was combined with other eye surgery. The main reasons for performing the procedures included the desire to improve vision and eye aesthetics. In most cases the author obtained the intended effects through the use of iris suture techniques, without the use of iris implants. Proper qualification for the procedure, implementation of a specific suture technique and the presence of a sufficient amount of iris tissue constitute an element necessary to treat iris defects without the need for implants.
\end{abstract}

Index Terms - Eye trauma, iris coloboma, iris defect, partial iris implants, surgical iris repair.

\section{INTRODUCTION}

Surgical iris repair constitutes one of the permanent elements of eye surgery. It is mainly used in the case of post-traumatic lesions, less often for congenital iris defects. Taking into account surgical treatment, iris defects can be treated in three ways, i.e. iris implants, iris suture techniques or both procedures combined [1,2,3,4,5]. In selected cases, the combination of both techniques gives the greatest benefits, combining functional and aesthetic effects [3]. Iris implants are used increasingly often due to their variety and easier access. The type of implant is adjusted to the size of the defect. There is a broad selection, ranging from partial implants to artificial iris implants, applicable in the case of significant damage or total aniridia. Unfortunately, many authors point out the complications associated with the use of implants; therefore, they should not be overused $[5,6,7]$. Iris suturing has become relatively easy thanks to the slipknot technique used $[8,9]$. Properly used suture technique allows for the correction of almost all small iris defects and reduction of the pupil diameter. The use of iris implants has an advantage allowing for the correction of all iris defects, including those that cannot be corrected using suture techniques. However, it cannot be said that this technique is better. Each insertion of an iris implant requires the removal of the patient's own lens, even if it is clear and has the accommodation ability. The choice of surgical

Published on June 14, 2020

A. Cywinski, Silesian Eye Treatment Centre, Poland.

(corresponding e-mail: adamcyw@gmail.com). technique is determined by the condition of the iris and the surgeon's skills. Treatment economics is important, however not decisive.

\section{MATERIAL AND METHODS}

Iris surgery is one of the services provided at the Silesian Eye Treatment Center (Zory, Poland) as part of eye reconstructive surgery. Between 2012 and 2019, fifty-three iris surgical procedures were performed in forty-seven people at the Center. Table I lists all the causes resulting in iris defects and thus qualification for the procedure.

TABLE I: REASONS OF IRIS DEFECTS

\begin{tabular}{lc}
\hline \hline Reasons of iris defects: & $\begin{array}{c}\text { Numer } \\
\text { patients (eyes) }\end{array}$ \\
\hline Eye trauma: & $28(29)$ \\
Blunt & $12(12)$ \\
Penetrating & $13(14)$ \\
Surgical & $3(3)$ \\
Congenital iris defects: & $11(16)$ \\
Partial iris loss (coloboma) & $8(11)$ \\
Aniridia & $1(2)$ \\
Congenital wide pupil & $1(2)$ \\
Iris fibers covering pupil center & $1(1)$ \\
Other reasons: & $8(8)$ \\
Postinflammatory damage of m. sphincter pupillae & $4(4)$ \\
Damage of m. sphincter pupillae after acute angle & $3(3)$ \\
closure glaucoma & \\
Embolia of arteria centralis of the retina & $1(1)$ \\
\hline \hline
\end{tabular}

Graphically, the percentage share of causes of iris defects is shown in Fig 1.

Iris defects and reasons for their appearance (\%)

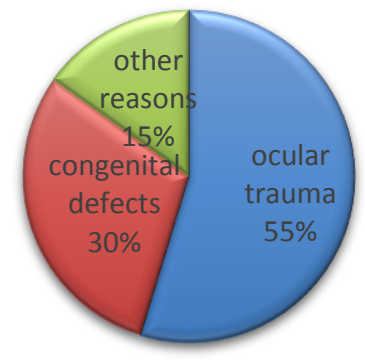

$\boldsymbol{\square}$ ocular trauma $\mathbf{\square}$ congenital defects $\boldsymbol{\square}$ other reasons

Fig. 1. Iris defects and reasons for their appearance (\%).

Broadly understood eye trauma is the main cause of iris damage. The next group included patients with congenital defects. Other reasons included post-inflammatory lesions, 
consequences of acute angle closure glaucoma and lesions caused by the embolia of arteria centralis of the retina. The procedure was performed in both eyes of six patients, while three patients had the procedure due to partial congenital iris defect (coloboma). Very wide pupils, congenital aniridia and penetrating eye trauma were the cause of surgery in both eyes in another three patients. The procedures were performed by one surgeon, Dr. Adam Cywinski. The main purpose of surgery was to improve the quality of vision and visual acuity. Another reason was the improvement in eye aesthetics. The average age of patients was 44, ranging from 16 to 87 . Overall, more than $54 \%$ of patients undergoing repair surgery were male. Given eye trauma, $82 \%$ of all cases were male.

\section{A. Blunt eye trauma}

This trauma, diagnosed in twelve people (eyes), was mainly the cause of total or partial damage to the sphincter muscle, resulting in permanent dilatation of the pupil. The consequences of blunt trauma and surgical procedures performed prior to consultation at the Center are listed in Table II.

TABLE II: BLunT TRAuma. REASONS OF IRIS DAMAGE AND SURGICAL PROCEDURES MADE BEFORE EXAMINATIONS

\begin{tabular}{|c|c|}
\hline $\begin{array}{l}\text { Blunt trauma. Reasons od iris damage and surgical } \\
\text { procedures made prior to reconstructive surgery } \\
\qquad(\ldots)\end{array}$ & Number of eyes \\
\hline $\begin{array}{l}\text { Total damage of } \mathrm{m} \text {. sphincter pupillae permanent } \\
\text { dilatation of the pupil }\end{array}$ & 9 \\
\hline Partial (2-4 hrs.) damage of $\mathrm{m}$ sphincter pupillae & 3 \\
\hline Posttraumatic cataract (subluxation of the lens) & $8(4)$ \\
\hline $\begin{array}{l}\text { Dislocation of the lens into the vitreous (posterior } \\
\text { vitrectomy) }\end{array}$ & $2(2)$ \\
\hline $\begin{array}{l}\text { Rupture of the eyeball in the limbus area with } \\
\text { needed surgical intervention }\end{array}$ & $1(1)$ \\
\hline Retinal detachment (posterior vitrectomy) & $2(2)$ \\
\hline Partial avulsion of the iris from the base & 2 \\
\hline RPE atrophy in posterior pole of the eye & 2 \\
\hline Choroidal rupture in posterior pole of the eye & 2 \\
\hline $\begin{array}{l}\text { Partial dislocation of artificial lens into the anterior } \\
\text { chamber }\end{array}$ & 1 \\
\hline
\end{tabular}

Repair surgery performed at the Center mainly involved combined procedures, i.e. cataract removal with artificial lens implantation and reduction of the pupil diameter.

In five eyes a continuous suture was applied on the margin due to complete dysfunction of the iris sphincter muscle. In the following three cases (eyes where iris sphincter was damaged at the range between 2-4 hours, simple stitches were placed, thus preserving the function of the sphincter. In addition, in the case of subluxation of the lens, a capsular tension ring was introduced (4 eyes), and in two cases anterior vitrectomy was performed. In the remaining four eyes after posterior vitrectomy, continuous suture was placed on the pupillary margin, however in two eyes with postoperative aphakia transscleral suture fixation of a monofocal artificial lens was performed. A monofocal Carlevale intraocular lens by Soleko is an implant preferred by the author. This lens does not require fixation due to the specific structure of the haptic parts (Fig. 2).

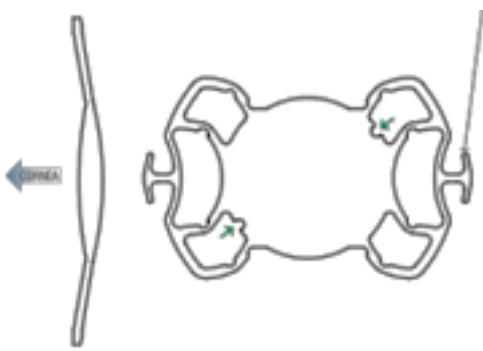

Fig. 2. Intraocular lens for transscleral fixation, Carlevale model by Soleko

The technique used to reduce the pupil diameter applying sutures is described in more detail in Iris plastic surgery. Part 1. Surgery without using of iris implants. A case series report [3]. In one eye, the procedure combining iris plastic surgery and implantation of an artificial lens were performed twice, which was dictated by the incorrect value of the artificial lens implanted during the first procedure. The procedure is described in detail in the following article: A surgical repair of the eye of 20 years old female after blunt trauma. A case report [10]. In the case of iridodialysis accompanying a significant dilatation of the pupil, in addition to the suture being placed on the pupillary margin, additional stitches were placed on the defect. Due to the absence of post-traumatic lesions within the cornea, the macula and the capsular bag, after cataract removal, an asymmetric bifocal lens with an addition of +3.0 by Oculentis (4 eyes) was implanted. In the remaining five cases, monofocal lenses were used. In one case, the procedure involving the displacement of the posterior chamber lens from the anterior chamber to the space behind the iris and reduction of the pupil size was performed 15 years after the trauma.

\section{B. Penetrating eye trauma}

This type of trauma was found in fourteen eyes in thirteen patients. The main causes of damage were trauma with a sharp tool or penetration of a foreign body inside the eye. In one case, the cause of iris defect was self-inflicted cutting the cornea of both eyes with a sharp tool) resulting in iridencleisis into the corneal wound. The causes and defects, as well as the procedures that were performed before the consultation at the Center are listed in Table III.

TABLE III: Penetrating Trauma. Reasons The Trauma, Its Consequences For The Eye And Surgical Procedures Made Prior TO RECONSTRUCTIVE SURGERY

\begin{tabular}{lc}
\hline \hline $\begin{array}{l}\text { Penetrating trauma. Reasons the trauma, its consequences } \\
\text { for the eye and surgical procedures made prior to } \\
\text { reconstructive surgery (...) }\end{array}$ & $\begin{array}{c}\text { Number of } \\
\text { eyes }\end{array}$ \\
\hline Penetrating trauma with sharp tool & 8 \\
Penetrating trauma with foreign intraocular body & 6 \\
Posttraumatic cataract (removal with IOL implantation) & $14(7)$ \\
Posttraumatic cataract (removal without IOL & $14(3)$ \\
implantation) & \\
Lens dislocation into vitreous (posterior vitrectomy) & 4 \\
Retinal detachment (posterior vitrectomy) & 4 \\
Vitreous hemorrhage (posterior vitrectomy) & 2 \\
Significant damage or total loss of the iris & 4 \\
Total damage of m. Sphincter pupillae & 10 \\
Perforating corneal wound (suture) & $11(11)$ \\
RPE atrophy in posterior segment of the eye & 4 \\
Secondary glaucoma (transscleral cyclophotocoagulation) & 3 \\
Partial dislocation of the artificial lens into anterior & 1 \\
chamber & \\
\hline
\end{tabular}


Repair procedures performed at the Center included: transscleral fixation of combined intraocular implant, correcting aniridia and aphakia (4 eyes) and the partial iris implant placement (2 eyes). Among others, Morcher models, types 67 and 68, were used. In two of the above cases, a continuous suture was placed on the margin of the significantly dilated pupil to improve the cosmetic effect. Due to this, the color of the iris of the treated eye did not differ from the color of the healthy eye.

Aniridia rings, type $96 \mathrm{~F}$ by Morcher, were used in the patient subjected to iridencleisis into the corneal wound. In the next four patients (4 eyes) transscleral fixation of an artificial lens was combined with a decrease in the pupil size was obtained by placing a continuous suture on its margin (3 eyes) or two stitches (1 eye).

A more detailed description of cases and the applied technique to correct defects using iris implants was provided in the publication entitled Iris plastic surgery. Part 2. Surgery with using of iris implants and combined techniques. Case reports. [4 In three patients the surgical procedure caused iris trauma. In one patient severe dilatation of the pupil and secondary adhesion of the iris to the cornea was caused by the endotamponade with expansile gas. A consequence of the gas expansion used during posterior vitrectomy resulted in the lens displacement towards the cornea. Treatment included surgical separation of the iris from the cornea and placement of a continuous suture on the pupillary margin to reduce the size of the pupil. In the next case, iridencleisis into the postoperative wound occurring during cataract surgery caused a significant peripheral pupil displacement. Because a change in the position of the head caused an improvement in vision, a combined procedure involving sewing of no reacting to light pupil and creating a new pupil in the central part of the iris, using a vitrectome. In the third case, huge iridectomy modo Ando caused a significant degree of vision loss, resulting in the effect of a second pupil. Placing one stitch on the iris removed this defect. A short video presenting the above procedures can be watched on the following website: https://medtube.pl/okulistyka/filmy-medyczne/24181-oneday-with-iris-plastic-surgery-posttraumatic-postsurgicalpostinflammatory-and-congenital-reasons-of-this-repairwill-be-presented, and

https://www.youtube.com/watch? $v=z o F V 4 O o W N U 4 \& t=9 s$.

\section{Congenital iris defects}

They mainly include partial defects, such as coloboma. Total aniridia and congenital, significant pupil dilatation were found in two patients, in both eyes. In the case of coloboma, the use of the author's proprietary surgical technique described in the article Iris plastic surgery in congenital coloboma modo Cywinski allowed for the defect to be closed without the adverse effect of the pupil displacement towards the periphery of the eye. This technique does not require the removal of the patient's lens, which is very important for patients, the majority of whom are of a young age. Bilateral implant of an artificial iris in the eyes with accompanying cataract and nystagmus is described in a separate article. Another patient treated at another eye center, despite the congenital pupil dilatation (8$9 \mathrm{~mm}$ in photopic conditions) was implanted an asymmetric bifocal intraocular lens. Such a large pupil diameter constitutes a contraindication to implanting premium class lenses. Instead of replacing the lens with a monofocal lens, it was decided to reduce the pupil diameter by applying simple stitches to the pupillary margin. Significant improvement in quality, preservation of distance vision and reading ability without additional correction caused that the patient decided to have a similar procedure performed in the other eye. After implantation of a toric, bifocal lens, four simple stitches were placed on the pupillary margin obtaining a diamond shape. The use of simple stitches in this case allowed for the effect of physiological change in the pupil size depending on light intensity to be preserved. Another interesting case was the presence of iris fibers, almost completely covering the pupil. Their removal not only improved the eye aesthetics, but above all improved visual acuity. A broader description of the above cases is included in the abovementioned publication [3].

\section{Other reasons of iris defects}

An acute angle closure glaucoma and recurrent iritis are other causes that have led to irreversible dilatation of the pupil. In five eyes a decrease in the pupil size was combined with cataract removal, of which bifocal lenses were implanted in three cases. In the eyes with artificial lenses (2), only a decrease in the pupil size was performed. In the above cases, a continuous suture was placed on the pupillary margin due to complete damage to the sphincter muscle. In the other two cases, where sphincter damage was only partial, simple stitches were applied at the damage sites. An interesting case concerned vision loss after embolia of arteria centralis of the retina. White intumescent cataract, severe dilatation of the pupil and a change in eye color as a consequence pigment deposits covering whole iris caused that the patient decided to have a procedure aimed at removing the cosmetic defect performed. The cataract was removed, the pupil was reduced by applying simple stitches, and the pigment epithelium was additionally removed from the surface of the iris. The patient regained her natural blue eye color [3]. In each of the above cases, the pupil size was determined comparatively, based on the diameter of the pupil of the healthy eye, examined under photopic conditions.

\section{RESULTS}

Out of fifty-three corrected iris defects, iris implants were used only in eight eyes. In other cases, only techniques of iris suture were used. In the case of a wide pupil and complete damage to the sphincter, a continuous suture was applied. If the sphincter damage was partial the defect was small, or the congenital defect was corrected, i.e. coloboma, simple stitches were applied.

\section{A. Photophobia and blurred vision}

They disappeared in almost every case, except for one in which there was a corneal edema increased. This patient, due to the presence of a post-traumatic scar, passing through the center of the cornea, was initially qualified for corneal transplantation. Repair procedure in this eye involved 
transscleral fixation of an artificial lens combined with the pupil diameter reduction (4 simple stitches).

\section{B. Subjective improvement of visual acuity.}

A beneficial effect was not obtained in two cases (eyes). One of them was described above, another was caused by endophthalmitis. In this patient, the trauma affected both eyes and was caused by self-infliction In the second eye, subjected to iris plastic surgery combined with a partial iris implant, both improved visual quality and visual acuity in distance and near vision were obtained. The author does not include cases of coloboma involving the macular area and eyes with diagnosed vision loss subjected to cosmetic surgery in the group of patients in whom the quality of vision and visual acuity did not improve.

\section{Cosmetic effect}

It was obtained in all cases, including eyes with vision loss and impaired vision. The exception were the two cases described above. The cosmetic effect included, among others, closing of the partial iris defect, pupil diameter reduction, restoration of the iris color, white eye effect removal (combination of cataract removal with pupil diameter reduction) and obtaining the patient's eye color after bilateral artificial iris implantation in a patient with congenital aniridia. As mentioned before, in patients who were implanted a black implant correcting aniridia and aphakia, applying continuous suture on the margin of a significantly dilated pupil allowed for a color similar to that of the iris of the healthy eye to be obtained.

\section{Pupil diameter}

In the eyes with iris plastic surgery, the pupil diameter was reduced by approx. $3.3 \mathrm{~mm}$ on average, from approx. 8 $\mathrm{mm}$ to $4.7 \mathrm{~mm}$. In the eyes with partial damage to the sphincter, thanks to the placement of simple stitches on the site of damage, the sphincter function was preserved. In the eyes with coloboma, the effect of an almost round pupil the effect of a pupil almost round in shape was obtained, which compared with the preoperative condition, when the shape of the pupil with the defect resembled a keyhole, gave a very good cosmetic effect.

\section{E. Visual acuity}

The study evaluated comparative values of visual acuity before and after surgery, for distance and near vision, with the best correction (BCVA). Unfortunately, some patients, before the repair procedure, were not subjected to any correction of the existing eye defect, despite aphakia, which was additionally accompanied by a very large pupil or its complete absence. In such cases, the greatest improvement in distance visual acuity, examined using Snellen charts, was obtained. The average preoperative value was 0.02 , in the range of: hand movements up to 0.06 , the postoperative value was up to 0.7 , in the range from 0.6 to 1.0 . In nine eyes (7 people), after cataract removal, an asymmetric bifocal lens with +3.0 Dsph addition (Mplus) was implanted, which allowed an average visual acuity value up to 0.9 distance vision, D-0.75 near vision to be obtained without additional correction. In cases of post-traumatic pupil damage, the use of an asymmetric intra-ocular lens (Mplus) allows for some independence from the size and function of the pupil. Comparison of average values of BCVA obtained preoperatively and 2-6 months after surgery is presented in Fig. 3.

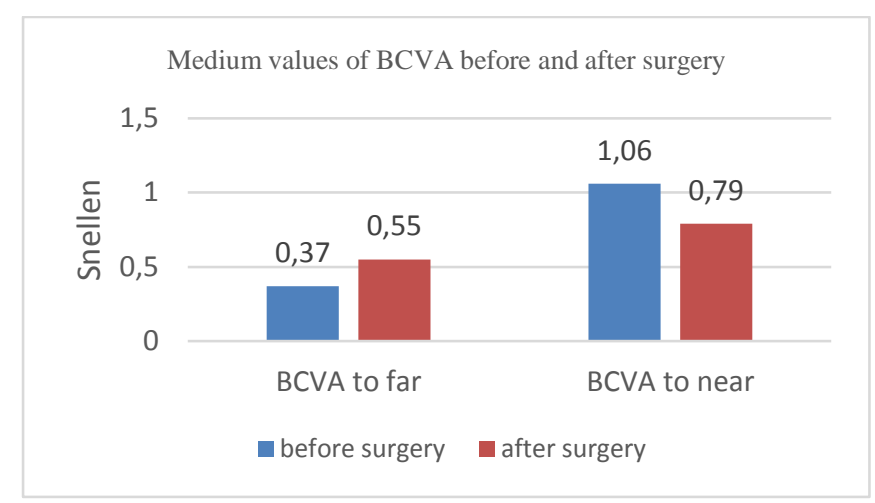

Fig. 3. Medium values of BCVA before and after surgery.

\section{F. Postoperative complications.}

All complications that were observed after the repair procedure are listed in Table IV.

TABLE IV: POSTOPERATIVE COMPLICATIONS

\begin{tabular}{lc}
\hline \hline Postoperative complications & $\begin{array}{c}\text { Number of } \\
\text { eyes }\end{array}$ \\
\hline $\begin{array}{l}\text { Transient hemorrhage ad anterior chamber } \\
\text { Transient hemorrhage ad vitreous cavity }\end{array}$ & 30 \\
\hline Transient increase of intraocular pressure & 15 \\
\hline Transient corneal edema & 11 \\
\hline $\begin{array}{l}\text { Secondary glaucoma treated with eye drops } \\
\text { application }\end{array}$ & 13 \\
\hline $\begin{array}{l}\text { Refractory glaucoma, needed Ahmed valve } \\
\text { application }\end{array}$ & 1 \\
\hline $\begin{array}{l}\text { Permanent corneal edema, needed corneal } \\
\text { transplantation }\end{array}$ & 1 \\
\hline Endophthalmitis & 1 \\
\hline $\begin{array}{l}\text { Postoperative eye defect - exchange of } \\
\text { artificial lens }\end{array}$ & 1 \\
\hline $\begin{array}{l}\text { Artificial lens calcifitation (factory defect) } \\
\text { Cataract remnants in vitreous cavity, needed } \\
\text { posterior vitrectomy }\end{array}$ & 1 \\
\hline $\begin{array}{l}\text { Subluxatio of partial aniridia implants into } \\
\text { anterior chamber }\end{array}$ & 2 \\
\hline \begin{tabular}{l} 
Glare effect after bifocal lens implantation \\
\hline
\end{tabular} & 1 \\
\hline
\end{tabular}

Transient hemorrhage into anterior chamber and vitreous did not require treatment. In the event of increased pressure, anti-glaucoma eye drops and dorzolamide used orally were recommended, usually for 3-4 days. Corneal edema was treated with hypertonic $5 \% \mathrm{NaCl}$ saline solution. Traumatic glaucoma was another consequence of the trauma. In one case with refractory glaucoma normalization of eye pressure was obtained only after Ahmed valve implantation made in the author's own modification [12]. In other cases, pressure normalized after using anti-glaucoma drops. The displacement of partial iris implants into the anterior chamber was caused by severe nystagmus. A detailed analysis of this case, carried out in a separate study, seeks to find a treatment option to avoid this complication. The glare effect was observed in two patients (eyes) after bifocal lens implantation, Mplus model, mainly under scotopic 
conditions. Considering the condition prior to cataract removal and iris plastic surgery, this effect was marginalized by patients. In cases of artificial lens calcification, despite cataract removal five years earlier, the lens was replaced after sutures reducing the pupil size were removed, and they were reapplied after the lens was inserted.

\section{CONCLUSIONS}

Currently, iris reconstructive surgery is developed to such an extent that it allows for the removal of the existing defects in almost every case. Using the appropriate technique of iris suturing and iris implants, it is possible not only to improve the quality of vision and visual acuity, but also correct the cosmetic defect. Most defects can be corrected using only iris suture techniques with minimal risk of postoperative complications. This also applies to congenital iris defects, such as coloboma, where using the author's proprietary suture technique may result in several benefits, including preservation of the patient's lens and preventing the pupil from its displacement towards the corrected defect. By avoiding the use of iris implants for any iris defect or sphincter damage resulting in permanent dilatation of the pupil, the risk of complications associated with the presence of a foreign body in the form of an iris implant is reduced. Using iris suture techniques, a natural material is used, i.e. the iris, a formation susceptible to stretching and to a large extent resistant to damage associated with the application of stitches. The combination of an iris implant with iris plastic surgery has a favorable cosmetic and economic dimension. Obtaining a similar visual effect with the use of an artificial iris significantly increases the cost of the procedure, which is of great significance in the sphere of economics. If the patient's lens needs to be removed, to avoid a postoperative defect, it is suggested to repeat the measurement of an artificial intraocular lens 2-3 times or to use two different methods of measuring it.

\section{REFERENCES}

[1] Hu S, Wang M, Xiao T, Zhao Z. Iris reconstruction combined with iris-claw intraocular lens implantation for the management of iris-lens injured patients. Indian J Ophthalmol 2016 Mar; 64(3): 216-221.

[2] Congenital iris coloboma repair using a modified McCannel suture technique. Blackmon DM, Lambert SR. Am J Ophthalmol. 2003 May;135(5):730-2.

[3] Cywinski A, Iris plastic surgery. Part 1. Surgery without iris implants. OphthaTherapy 2019; 4(24): 265-271.

[4] Cywinski A. Iris plastic surgery. Part 2. Surgery with using of iris implants and combined techniques. Case reports. OphthaTherapy 2020; 1(25): 59-65.

[5] Galvis V., Tello A., Corrales M.I. Postoperative results of cosmetic iris implants. J Cataract Refract Surg 2016; 42:1518-1526.

[6] Complications of cosmetic iris implants: French series of 87 eyes. El Chehab H, Gatinel D, Baudouin C, Muraine M, Hoffart et al. Cataract Refract Surg. 2020 Jan;46(1):34-39.

[7] Chaurasia S, Devastating complication of cosmetic iris implants Indian J Ophthalmol 2017 Aug; 65(8): 771-772.

[8] Siepser S.B. The closed chamber slipping suture technique for iris repair. Ann Ophthalmol. 1994;26(3):71-72.

[9] Chang D.F. Siepser slipknot for McCannel iris- suture fixation of subluxated intraocular lenses. J Cataract Refract Surg. 2004;30: $1170-1176$.

[10] Cywinski A. Surgical repair of an eye of 20 years old female after blunt trauma. A case report Okulistyka po Dyplomie 2019, 06

[11] Cywinski A., Ferda Lewinska D. Iris plastic surgery in congenital coloboma modo Cywinski. OphthaTherapy 2018; 1(17): 46-51.
[12] Cywinski A., Bednarski L. A Treatment of Refractory Glaucoma in Pseudophakic Patients after Posterior Vitrectomy using the Ahmed Valve in an Own Modification. International Journal of Research Studies in Medical and Health Sciences Volume 4, Issue 6, 2019, PP 01-05.

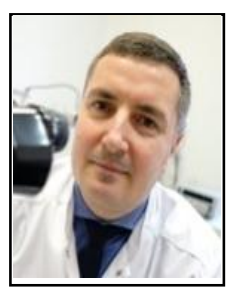

Adam Cywinski, date of birth 22.09.1967. Medica Director and founder of private medical center, Silesian Eye Treatment Centre, Zory, Poland. Consultant of posterior segment diseases. He graduated Silesian Medical Academy in Katowice and gained medical degree in the biggest Clinic of Ophthalmology, also in Katowice. He doctorated in surgical topic "Posterior vitrectomy with gas endotamponade and ILM peeling in IV stage macular hole, in 2003. As a surgeon he made a few thousands posterior vitrectomy procedures, including macular diseases, retinal detachment, diabetic and posttraumatic cases. His next interest is anterior segment surgery with special focus on reconstruction of the iris. His everyday job includes cataract surgery of complicated cases and surgery with premium class lenses. Dr Cywinski is a member of PTO, AAO, ESCRS, Euretinan. 